\title{
Conflictos bioéticos en la formación de profesionales con discapacidad
}

\author{
Verónica Teresa Guerra Guerrero \\ Universidad Católica del Maule, Talca, Chile.
}

\section{Resumen}

La discapacidad presenta un incremento significativo en todo el mundo, afectando principalmente a poblaciones vulnerables, como la de los jóvenes, que presentan peores resultados académicos y de salud en comparación con personas sin discapacidad. El objetivo del artículo es analizar los conflictos éticos que subyacen a la formación de estudiantes con discapacidad del área de la salud, particularmente en enfermería, que realizan prácticas en escenarios clínicos en donde los usuarios requieren cuidados de calidad. Como metodología se propone el análisis bioético de una situación de conflicto. El artigo concluye que es necesario desarrollar políticas que incluyan a los estudiantes con discapacidad en la educación superior.

Palabras clave: Personas con discapacidad. Bioética. Educación superior. Estudiantes. Estudiantes de enfermería.

\section{Resumo}

\section{Conflitos bioéticos na formação de profissionais com deficiência}

A deficiência apresenta um aumento significativo em todo o mundo, afetando principalmente populações vulneráveis, entre as quais se destacam jovens que apresentam os piores resultados acadêmicos e de saúde, em comparação a pessoas sem deficiência. Existem convenções internacionais que orientam a inclusão de estudantes com deficiência nos campos acadêmico e curricular. O objetivo deste artigo é analisar os conflitos éticos subjacentes à formação de profissionais do ensino superior portadores de deficiência, que estudam na área da saúde, particularmente na área de enfermagem, e trabalham em ambientes clínicos onde os usuários necessitam de cuidados de qualidade. Como metodologia, propõe-se a análise bioética com foco na revisão dos antecedentes da deficiência - situação de conflito bioético - e a proposta de enfrentar a situação de conflito segundo o discernimento bioético. São necessários esforços importantes ao nível das políticas educacionais que incentivam a inclusão dos estudantes com deficiência no ensino superior.

Palavras-chave: Pessoas com deficiência. Bioética. Educação superior. Estudantes. Estudantes de enfermagem.

\begin{abstract}
Bioethical conflicts in the training of professionals with disabilities

Disability presents a significant increase worldwide, mainly affecting vulnerable populations, such as young people, who have worse academic and health outcomes, compared with able bodied peope. There are international conventions that guide the inclusion of students with disabilities, at the academic level and curriculum. This article analyzes the ethical conflicts that underlie the higher education training of professionals with disabilities in the area of health, particularly nursing, and who work in clinical environments where users require care quality. As a methodology, the bioethical analysis is proposed focused on the background review on disability - situation of bioethical conflict and the proposal to address the conflict situation according to bioethical discernment. Important efforts are required at the level of educational policies to properly include students with disabilities at the higher education.
\end{abstract}

Keywords: Disabled persons. Bioethics. Higher education. Students. Nursing students. 
Según el Informe Mundial sobre la Discapacidad del 2011, existen más de mil millones de personas en el mundo que viven con algún tipo de discapacidad, lo que significa alrededor del $15 \%$ de la población global ${ }^{1,2}$. En este informe la Organización Mundial de la Salud (OMS) ${ }^{3}$ señala además que la discapacidad afecta principalmente a poblaciones vulnerables, siendo más común en mujeres, personas mayores, niños y adultos pobres.

Según la $\mathrm{OMS}^{3}$, las personas con discapacidad presentan resultados de salud y académicos peores en comparación con las personas sin discapacidad. Adicionalmente, tienen una menor participación económica y tasas de pobreza más altas. Estos resultados son atribuidos a las dificultades en el acceso a servicios básicos como salud, educación, empleo, transporte e información (dificultades que se acentúan particularmente en poblaciones menos favorecidas).

La discapacidad es una condición que experimenta el ser humano en forma compleja, por lo que el desarrollo de intervenciones para superar las desventajas son múltiples, sistémicas y varían según el contexto ${ }^{3}$. Al respecto, la Convención sobre los Derechos de las Personas con Discapacidad (CDPD), aprobada por la Organización de las Naciones Unidas (ONU)en el 2006, pretende promover, proteger y asegurar el goce pleno y en condiciones de igualdad de todos los derechos humanos y libertades fundamentales por todas las personas con discapacidad, y promover el respeto de su dignidad inherente ${ }^{4}$. La discapacidad forma parte de la condición humana, y la mayoría de las personas pueden llegar a sufrir algún tipo de discapacidad transitoria o permanente en algún momento de su vida, convirtiéndose en personas vulnerables y con necesidades especiales ${ }^{3}$.

La vulnerabilidad y dignidad que las Naciones Unidas propone considerar en las personas con discapacidad son aspectos que también han sido descritos como principios de la bioética ${ }^{5}$. Autores como Torralba Roselló ${ }^{6}$ señalan que la vulnerabilidad es el principio más vinculado con la fragilidad de las personas. Un ser humano vulnerable, tal como señala el autor, es un ser quebradizo, cuya integridad puede estar amenazada constantemente por razones externas e internas. Una persona con discapacidad se convierte en un ser vulnerable, dependiente y limitado en sus acciones, sin posibilidad de ser absoluto y autosuficiente.
Bajo esa condición, las personas vulnerables se ven afectadas en todas las dimensiones de su vida.

Respecto a la dignidad, Torralba Roselló ${ }^{6}$ la define como un atributo o característica de la persona y señala que reconocerla implica relacionarla con el respeto. Se entiende que la dignidad no es un atributo de tipo físico o natural, sino algo que se predica desde el ser, por el solo hecho de ser una persona. La dignidad es parte de la condición humana y, por lo tanto, afirmar la dignidad de una persona significa que no se puede atentar contra ella, ni tratarla de manera inferior a su categoría ontológica? ${ }^{7}$.

Considerando lo anterior y que la discapacidad es una condición en que las personas experimentan vulnerabilidad, pérdida de autonomía y de su integridad, es imperativo crear ambientes que reduzcan esas condiciones y promuevan la dignidad de las personas como un derecho humano esencial. Mediante el método de discernimiento bioético, este artículo tiene como objetivo analizar los conflictos éticos que subyacen a la formación de estudiantes con discapacidad en el área de la salud, particularmente en enfermería, y que realizan prácticas en escenarios clínicos en donde los usuarios requieren cuidados de calidad.

El artículo se divide en tres secciones: una primera sección en la que se describen los antecedentes y principales conceptos que involucra la discapacidad en la educación superior; una segunda sección que aborda una situación de conflicto en el ámbito académico -específicamente en la carrera de enfermería- que requiere un abordaje bioético; y una tercera sección con una propuesta para solucionar la situación de conflicto utilizando el discernimiento bioético.

\section{Antecedentes sobre discapacidad en la educación superior}

\section{Discapacidad en el mundo y en Chile}

La Clasificación Internacional del Funcionamiento, de la Discapacidad y de la Salud (CIF) define la discapacidad como un término genérico que engloba deficiencias, limitaciones de actividad y restricciones a la participación ${ }^{3}$. La discapacidad, según esta definición, denota aspectos negativos que ocurren en la interacción entre personas que tienen un problema de salud 
(como parálisis cerebral, síndrome de Down o depresión) y factores personales y ambientales (como actitudes negativas, transporte y edificios públicos inaccesibles o falta de apoyo social).

Según el Informe Mundial de la $\mathrm{OMS}^{3}$, el número de personas con discapacidad está creciendo, lo que se debe principalmente al envejecimiento de la población, puesto que las personas ancianas tienen un mayor riesgo de discapacidad conforme ocurren cambios físicos, biológicos, psicológicos y económicos, entre otros. Sin embargo, las características de la discapacidad varían de un país a otro y están influenciadas por los propios problemas de salud, como accidentes de tránsito, cáncer, enfermedades crónicas y cardíacas, conflictos, hábitos alimentarios y abuso de sustancias. Respecto a los problemas de salud, los accidentes de tránsito derivan en algunos casos en discapacidad física, principalmente en personas jóvenes o adultos jóvenes. Este grupo etario es el que presenta mayor riesgo de sufrir este tipo de lesión, a diferencia de personas mayores, que enfrentan dificultades auditivas, visuales y físicas.

El informe de la OMS plantea, además, que existen visiones estereotipadas sobre la discapacidad, como las que insisten en los usuarios de silla de ruedas y en algunos otros grupos "clásicos" como personas ciegas o sordas ${ }^{8}$. Es así como se describe que personas con deficiencias físicas experimentan desventajas con mayor frecuencia, revelando una condición de inferioridad que afecta la interacción social y el desempeño de roles sociales.

Entre las principales dificultades que experimentan las personas con discapacidad, se destacan políticas y normas insuficientes, actitudes negativas por parte de la sociedad, problemas con la prestación de servicios, financiamiento insuficiente, falta de accesibilidad, falta de consulta y participación social y falta de datos y pruebas sobre la discapacidad $^{2,3}$, barreras que contribuyen a la exclusión social. En algunos casos, las oportunidades de acceso a los servicios solo aparecen cuando las personas con discapacidad insistentemente las demandan, amparadas en la legislación de su país.

Como resultado de esas dificultades, las personas con discapacidad se ven afectadas en los resultados en salud, en el ámbito académico y en la participación económica, presentando mayores tasas de pobreza, mayor dependencia y menor participación social ${ }^{9}$. En relación con los resultados académicos, se describe que los niños con discapacidad tienen menor probabilidad de ingresar a la educación escolar y permanecer en el sistema. El fracaso escolar se encuentra presente en todos los grupos de edad y países, pero sobre todo en países de ingresos bajos ${ }^{3}$.

La discapacidad involucra aspectos complejos, que no solo producen barreras físicas, sino también sociales, por lo que es responsabilidad de la sociedad proporcionar condiciones para superar estas barreras ${ }^{10}$. Así el presente artículo se basa en el modelo social de la discapacidad ${ }^{11,12}$, que, más allá de la funcionalidad, considera las limitaciones que la propia sociedad impone a la persona con discapacidad $^{13}$. Desde esta perspectiva, la discapacidad se define como la desventaja o restricción de actividad, causada por la organización social contemporánea que no considera, o considera en forma insuficiente, a las personas que tienen diversidades funcionales, y por ello las excluye de la participación en las actividades corrientes de la sociedad ${ }^{14}$.

Hay documentos que proponen derechos específicos para las personas con discapacidad, entre los que se destacan las Normas Uniformes sobre la Igualdad de Oportunidades para las Personas con Discapacidad de 1993, mediante el cual la ONU asumió la obligación de velar por los derechos de las personas con discapacidad ${ }^{15}$. Por su parte, los participantes de la Conferencia sobre Necesidades Educativas Especiales: Acceso y Calidad, realizada en 1994 en España, asumieron la tarea de visibilizar las necesidades educativas especiales. $Y$ en Chile, en 1994, el Congreso Nacional dictó la Ley $19.284^{16}$, que estableció normas para la plena integración social de personas con discapacidad ${ }^{10}$.

En el 2006, la ONU aprobó la Convención sobre los Derechos de las Personas con Discapacidad ${ }^{17}$, amparada en los derechos humanos y concebida como un instrumento de desarrollo social de los Estados signatarios, que se comprometieron a luchar contra los prejuicios y estereotipos y promover la conciencia acerca de las capacidades de las personas con deficiencias ${ }^{2}$. Entre los principios de esta convención se destacan: a) el respeto de la dignidad inherente, la autonomía individual, incluida la libertad de tomar las propias decisiones, y la independencia de las personas; b) la no discriminación; c) la participación e inclusión plenas y efectivas en la sociedad; d) el respeto por la diferencia y la aceptación de las personas con 
discapacidad como parte de la diversidad y la condición humanas; e) la igualdad de oportunidades; f) la accesibilidad ${ }^{18}$. Con base en esto se puede establecer que el modelo social es consistente con los valores que sustentan a los derechos humanos: la dignidad, la libertad entendida como autonomía y la igualdad de todos los seres humanos ${ }^{15}$.

Con respecto a los aspectos vinculados a la educación y discapacidad, tema de este artículo, la convención señala que los Estados partes asegurarán que las personas con discapacidad tengan acceso general a la educación superior, la formación profesional, la educación para adultos y el aprendizaje durante toda la vida, sin discriminación y en igualdad de condiciones con los demás. A tal fin, los Estados partes asegurarán que se realicen ajustes razonables para las personas con discapacidad ${ }^{19}$. Dichos ajustes consideran la incorporación de innovaciones, adecuaciones curriculares e infraestructurales, así como capacitación y sensibilización para la educación inclusiva ${ }^{2,10,15}$. En este sentido, en países como España, hubo un significativo avance en temas de inclusión educativa ${ }^{20}$. Lo mismo ha ocurrido en algunos países de Latinoamérica e donde los esfuerzos por alcanzar la inclusión en la educación superior se enfocan en analizar las condiciones y desarrollar acciones concretas ${ }^{20,21}$.

En el 2010, se aprobó en Chile la actual Ley $20.422^{22}$, que establece las Normas sobre Igualdad de Oportunidades e Inclusión Social de Personas con Discapacidad y crea el Servicio Nacional de la Discapacidad (Senadis), dependiente del Ministerio de Desarrollo Social. Con base en esta ley, también se elaboró la Política Nacional para la Inclusión Social de las Personas con Discapacidad (2013-2020) ${ }^{23}$. En su artículo $5^{\circ}$. La Ley 20.422 define la persona con discapacidad como aquella que teniendo una o más deficiencias físicas, mentales, sea por causa psíquica o intelectual, o sensorial, de carácter temporal o permanente, al interactuar con diversas barreras presentes en el entorno, ve impedida o restringida su participación plena y efectiva en la sociedad, en igualdad de condiciones con las demás ${ }^{22}$.

\section{Discapacidad en la educación de Chile}

En Chile, el Segundo Estudio Nacional de Discapacidad, auspiciado por el Banco Mundial y la OMS, realizado en el 2015, reveló que el 20\% de la población de 18 años o más (2.606.914 personas) se encuentra en situación de discapacidad ${ }^{10}$. Respecto al nivel de escolaridad, el informe mostró que el 7,4\% de las personas adultas en situación de discapacidad no tiene educación formal, el 23,4\% tienen escolaridad básica incompleta, el 16,1\% escolaridad básica completa, el 14,7\% enseñanza media incompleta, el 23,4\% enseñanza media completa, el $5,9 \%$ educación superior incompleta y solo el 9,1\% cuenta con educación superior completa ${ }^{10}$.

En la actualidad, la legislación chilena, mediante la Ley 20.422 , señala que se debe velar por el acceso de las personas con discapacidad a un sistema educativo inclusivo y con igualdad de oportunidades en todos sus niveles, desde la Educación Parvularia a la Educación Superior, caracterizado por la incorporación de innovaciones y adecuaciones curriculares y de infraestructura, con personal docente sensibilizado, capacitado y formado profesionalmente en la educación inclusiva. De igual forma, se deberá considerar la variable discapacidad en los sistemas e instrumentos de monitoreo periódico orientados a mejorar los estándares de calidad del sistema educacional discapacidad ${ }^{22}$.

Respecto a la educación superior, en su artículo 39, la ley establece que las instituciones de educación superior deberán contar con mecanismos que faciliten el acceso de las personas con discapacidad, así como adaptar los materiales de estudio y medios de enseñanza para que dichas personas puedan cursar las diferentes carreras discapacidad ${ }^{22}$.

En el 2011, un estudio del Ministerio de Educación mostró que, de un total de 174 instituciones preguntadas acerca de si poseían sistemas de adaptación para recibir alumnos en situación de discapacidad, solo 75 instituciones contestaron la encuesta, y de estas respondieron afirmativamente 15 universidades del Consejo de Rectores de las Universidades Chilenas (de un total de 25), 16 universidades privadas (35 en total), 15 institutos profesionales ( 43 en total) y 15 centros de formación técnica ( 75 en total) ${ }^{10}$. Respecto a los estudiantes con discapacidad, 637 estudiaban en las universidades del Consejo de Rectores (256 con discapacidad motora, 210 visual y 43 auditiva), 92 en universidades privadas, 89 en institutos profesionales y 25 en centros de formación técnica. Sus principales áreas de estudio fueron humanidades y salud, desglosándose en pedagogía, trabajo social, psicología, derecho y enfermería ${ }^{10}$. 
Inclusión de personas con discapacidad en la formación en enfermería

En la educación superior en enfermería hay una diversidad de perspectivas respecto a la inclusión. Por una parte, aunque existe un incremento en la prevalencia de la discapacidad, esta condición recibe escasa atención en la formación disciplinar ${ }^{24}$. Por otra parte, la temática de la discapacidad en estudiantes de enfermería ha sido desde décadas un tema de interés ${ }^{25,26}$. Ya en el 2004, un estudio señalaba que el número de estudiantes con discapacidad en los programas de enfermería en la educación superior de Estados Unidos se estaba incrementando ${ }^{25}$. Dicho estudio destacaba la obligación ética y legal de educar a los individuos que presentan discapacidad, señalando que la aceptación de ellos en los programas de estudios se intensificó después de 1990, con la aprobación de una la ley sobre el tema en el país. No obstante, los estudiantes de enfermería con discapacidad siguen enfrentando barreras como estereotipos y discriminación, puesto que muchas veces son vistos como profesionales sin las capacidades necesarias para proporcionar cuidado seguro, completo y de calidad, de acuerdo con las necesidades que puedan tener los pacientes ${ }^{25,27,28}$.

Internacionalmente se describe que las escuelas de enfermería tienen escasa experiencia en la incorporación de estudiantes que presentan discapacidad ${ }^{25}$, no obstante existe disposición positiva para recibirlos ${ }^{29}$. Esta situación ocurre también en Chile, donde, si bien la legislación promueve la inclusión de los estudiantes con discapacidad en la educación superior, la realidad muestra que todavía se requieren ajustes para la completa inclusión, mediante la creación de espacios y oportunidades para los jóvenes que desean estudiar.

Cambiar los programas de estudio es una necesidad real, y este cambio incluye modificar las políticas institucionales, formar equipos especiales, proporcionar tutores y reformar las dependencias de las instituciones ${ }^{25,30,31}$. Las personas con discapacidad pueden realizar las mismas tareas que las personas sin discapacidad, pero utilizando métodos diferentes a los tradicionales, lo que implica ajustes institucionales. La adaptación del currículo de enfermería podría ser considerada la base para facilitar el acceso y ayudar a integrar a las personas con discapacidad en la profesión ${ }^{25}$. En este sentido, un ejemplo positivo es el de la Organización Nacional de Enfermeras con Discapacidad, en Estados Unidos. La entidad apoya la recomendación de aceptar más personas con discapacidad para que estudien enfermería y al mismo tiempo lucha para que el criterio de habilidades funcionales, utilizado para el ingreso en las escuelas de enfermería, sea eliminado o modificado, una vez que ese criterio restringe las posibilidades de acceso ${ }^{31}$.

La inclusión tiene como base ofrecer educación a los estudiantes indistintamente, proporcionándoles una educación capaz de identificar las necesidades de todos (respecto a si ellos tienen deterioro, discapacidad o requieren una adaptación curricular), de modo que puedan aprender y desarrollarse como persona ${ }^{26,29-31}$. Algunos autores señalan que estrategias como la preparación de profesores y la existencia de tutores son fundamentales en la educación inclusiva ${ }^{27,29}$. Se plantea, además, que actitudes positivas respecto a los estudiantes con discapacidad son una importante cualidad de los profesionales, junto con la responsabilidad ética. Se recomienda que el equipo de profesionales promueva las relaciones de cooperación entre estudiantes con y sin discapacidad, facilitando la interacción entre ellos que debe ser simplificada por los profesores ${ }^{27}$.

La inclusión de estudiantes con discapacidad en las escuelas de enfermería es un proceso que debe ocurrir intencionadamente para promover el desarrollo de la comunidad universitaria ${ }^{28,31}$. El objetivo es favorecer las condiciones de educación de quienes son más vulnerables, en consideración a que las personas con discapacidad, tal como plantea Foucault, siguen siendo consideradas "anormales" 32.

\section{Perspectiva bioética del conflicto ético en el ámbito académico}

En el contexto clínico, se ha planteado que existen diversas formas de abordar las temáticas propias de la bioética. Al respecto, se describe que las propuestas de metodología en bioética o en ética clínica se basan en procesos que se desarrollan principalmente en dos fases o momentos: el análisis de los hechos y la fase normativa, o de decisiones ${ }^{33}$. Se destacan los análisis de caso de autores como Beauchamp y Childress, quienes 
abordan dilemas éticos mediante la aplicación de cuatro principios de la ética biomédica (autonomía, no maleficencia, beneficencia y justicia).

El método deliberativo que propone Diego Gracia, mediante el análisis de valores y la definición de posibles cursos de acción, también permite abordar situaciones de conflicto ético ${ }^{5}$. Por su parte, autores como Beca Infante ${ }^{33}$ proponen métodos para el análisis ético que sintetizan otras metodologías, como las de Diego Gracia y Albert Jonsen, con el fin de sistematizar los hechos y los valores, deliberar los cursos de acción y definir deberes. El autor establece como marco ético la dignidad del ser humano, teniendo presente las circunstancias y las consecuencias de las decisiones. El método incluye también los cuatro principios de la ética biomédica según la jerarquía planteada por Diego Gracia, que considera los principios de no maleficencia y justicia como de primer orden, y de segundo orden los principios de autonomía y beneficencia.

\section{Conflicto ético en el ámbito académico}

La dificultad enfrentada por los estudiantes con discapacidad en el desarrollo de actividades que requieren movimiento o desplazamiento puede ser considerada una situación de conflicto ético en el ámbito académico. Los estudiantes con discapacidades físicas permanentes en algunos casos encuentran obstáculos para realizar actividades básicas vinculadas con el movimiento, lo que puede generar dependencia de otras personas para cumplir con las responsabilidades académicas. Discapacidades que se generan durante el proceso de formación (como puede ocurrir con jóvenes que ingresan a estudiar sin discapacidad y esta se presenta en la etapa universitaria) también son situaciones que necesitan ser abordadas, considerando la perspectiva de la bioética.

Algunos países establecen protocolos de inclusión con mecanismos para facilitar la participación de las personas con discapacidad como estudiantes en formación. Dichos protocolos permiten que los estudiantes ingresen, progresen y finalicen sus estudios. Sin embargo, en las escuelas de enfermería, también hay situaciones más complejas de abordar, particularmente cuando los estudiantes tienen que realizar prácticas clínicas que involucran otras personas o pacientes. Puede ser difícil realizar los procedimientos de la manera como exigen la formación del profesional enfermero y las personas que necesitan de cuidados o atención en salud.

Situaciones en las que existe dificultad para que los estudiantes con discapacidad física desarrollen sus prácticas clínicas derivan en la necesidad de revisar y evaluar los currículos de las carreras de enfermería, donde los estudiantes tienen que lograr competencias declaradas en sus perfiles de egreso, vinculadas con el saber (cognitivas), el saber ser (conductual) y el saber hacer (procedimental). Las exigencias de la formación del profesional de enfermería se producen principalmente en escenarios de prácticas clínicas con la comunidad, en el hospital, y son requisitos que todo estudiante debe cumplir para alcanzar el título profesional. Sin embargo, se plantea un conflicto ético cuando se exige de los estudiantes con discapacidad que desarrollen las actividades vinculadas con el cuidado al igual que estudiantes sin discapacidad, como requisito para alcanzar el título profesional.

Derivado de lo anterior, se plantea además otro conflicto ético, puesto que los pacientes necesitan recibir cuidados de calidad, tanto por parte de los profesionales de la salud como de los estudiantes de enfermería. Al respecto, la legislación establece que los pacientes tienen derecho a exigir calidad en los cuidados y servicios que reciben en el sistema de salud. Es así como los estudiantes deben otorgar cuidados integrales, según las necesidades que tengan los pacientes, mediante la ejecución de procedimientos de enfermería adecuados y ajustados, tales como la administración de medicamentos, manejo de heridas y control de monitores.

\section{Propuesta de abordaje}

Con base en lo propuesto por Beca Infante ${ }^{33}$, los dos conflictos éticos descritos en la sección anterior se abordan con el siguiente esquema: 1) descripción del problema ético principal (descripción de los hechos que involucra); 2) análisis de los valores y principios éticos involucrados; y 3) identificación de los cursos posibles de acción, fundamentos y recomendación. 


\section{Descripción del problema ético principal}

El principal problema ético que existe en la situación anterior, que es objetivo de análisis en este artículo, es el siguiente: ¿Es posible que las instituciones de educación superior y que las Escuelas de Enfermería puedan ofrecer a los estudiantes que tienen discapacidad física la posibilidad de desarrollar sus estudios y terminar su formación, de modo de cumplir con las competencias esperadas en un profesional de la salud, particularmente un(a) enfermero(a), quien es responsable de otorgar cuidados oportunos y de calidad, según las necesidades individuales que tienen y podrían tener los pacientes del sistema de salud?

\section{Análisis de los valores y principios éticos involucrados}

Identificar las principales categorías bioéticas ${ }^{34-36}$ involucradas en el conflicto ético descrito requiere considerar tres perspectivas. En primer lugar, la perspectiva de los estudiantes con discapacidad física, que desean estudiar y finalizar sus estudios, y que son ciudadanos con derecho a educarse, incluye los principios bioéticos de la autonomía y de la justicia. En segundo lugar, la perspectiva de las instituciones de educación superior, en específico escuelas de enfermería, que tienen el deber ético y la responsabilidad de formar a un profesional competente para satisfacer las necesidades de salud de las personas, incluye los principios éticos de no maleficencia y beneficencia. En tercer lugar, la perspectiva de los usuarios del sistema de salud, quienes tienen el derecho a recibir atención de profesionales competentes, que brinden cuidados de salud de calidad, considera los principios éticos de justicia, no maleficencia y beneficencia.

Según lo anterior y el método de análisis ético-clínico propuesto ${ }^{33}$, los valores identificados en la situación de conflicto ético se describen en la Figura 1. Estos valores fueron identificados considerando el caso particular y la percepción personal de la autora, desde su perspectiva como académica de una institución de educación superior y profesional del área de la salud. Con base en la propuesta de análisis ${ }^{33}$, se consideran diversos tipos de valores: físicos, espirituales, estéticos, intelectuales, afectivos, económicos, políticos y religiosos, entre otros, los cuales se interrelacionan con el caso analizado. Derivan de los valores identificados en la Figura 1 los principios de justicia y de no maleficencia, puesto que los estudiantes tienen el derecho a que se les trate justamente, así como el derecho a recibir, por parte de la institución de educación superior, el mismo trato que reciben otros estudiantes sin discapacidad, siendo tratados de acuerdo con las políticas públicas nacionales e internacionales ${ }^{15}$. Adicionalmente, tienen el derecho a terminar sus estudios superiores, independiente de la discapacidad física que presenten. Sin embargo, este principio se contrapone al de no maleficencia, ya que el usuario o paciente también tiene el derecho a recibir atención por parte de un profesional que le brinde seguridad y calidad en la atención y le garantice atención libre de daño físico.

Del mismo modo, el principio de autonomía también se contrapone con el de no maleficencia, puesto que los estudiantes tienen derecho a terminar sus estudios con autonomía, pero esto no siempre puede ser asegurado por parte de las instituciones de educación superior, ya que los estudiantes con discapacidad física presentan limitaciones para acceder directamente a los pacientes, desplazarse independientemente y realizar procedimientos necesarios. La institución debe asegurar que los estudiantes efectivamente logren competencias mínimas que les permitan otorgar cuidados de calidad a los pacientes, libre de daños. 
Figura 1. Identificación de valores en conflicto ético de análisis

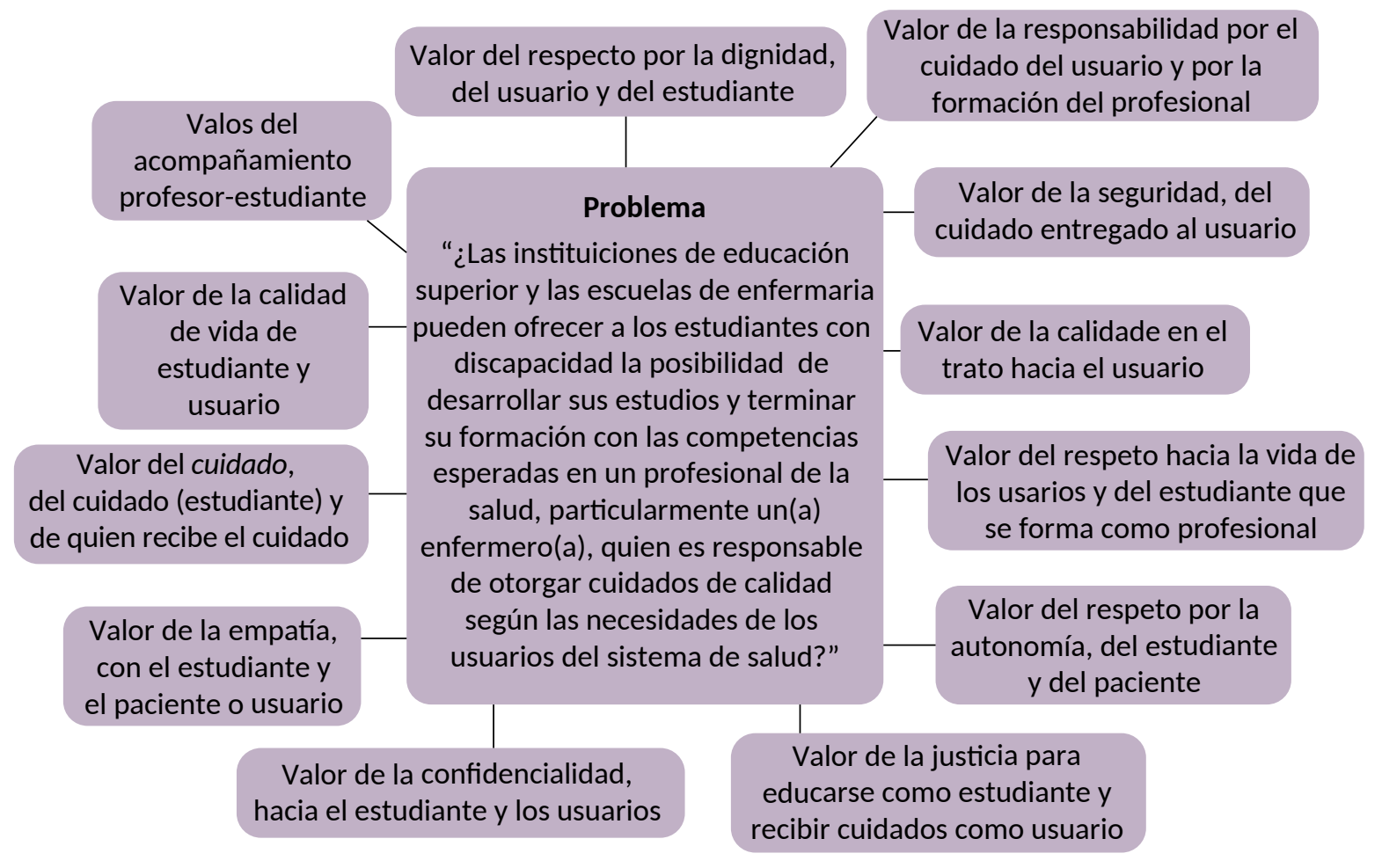

Cursos de acción posibles, fundamentos y recomendación

\section{Identificación de los cursos de acción posibles}

Primero: considerando los principios de justicia y autonomía, y que la inclusión es parte de los derechos humanos, las instituciones de educación superior y las escuelas de enfermería deberían realizar todas las adecuaciones curriculares que permitan a los estudiantes con discapacidad física formarse y ejercer la profesión ${ }^{15}$.

Segundo: considerando el principio de no maleficencia, las instituciones universitarias y escuelas de enfermería podrían restringir o limitar el acceso a la carrera de salud, puesto que los estudiantes con discapacidad pueden no cumplir con el perfil de egreso o no desarrollar las competencias procedimentales del saber hacer junto a las otras. Sin embargo, este curso de acción no cumple con las expectativas y definiciones que establecen las convenciones internacionales sobre inclusión.
Tercero: en relación con el conflicto ético analizado y considerando el principio de no maleficencia, las instituciones de educación superior y las escuelas de enfermería deberían realizar todas las adecuaciones curriculares que permitan desarrollar la formación profesional conforme el perfil del estudiante con discapacidad, asegurando al mismo tiempo las competencias necesarias y la verdadera inclusión.

Cuarto: en virtud del principio de justicia, es importante que la institución de educación superior se responsabilice y proteja el derecho que tienen los estudiantes con discapacidad de recibir un trato justo y en igualdad de derechos, particularmente para promover el principio de autonomía y cumplir con los cambios que se requieren no desde las personas, sino desde la sociedad, formando a los estudiantes sin dejar de considerar sus diferencias.

Considerando los cuatro cursos de acción, en este artículo se propone que el tercer y el cuarto curso son los más adecuados para resolver el conflicto ético descrito. 


\section{Consistencia del curso de acción elegido}

El curso de acción definido para resolver la situación de conflicto ético es respaldado por documentos de organizaciones internacionales y por la legislación chilena ${ }^{3,7,12}$. Por lo tanto, se trata de una recomendación que, además de ética, puede ser considerada un imperativo legal, lo que permite que sea defendida públicamente, en caso de ser requerido. Asimismo, la acción elegida, después de aplicada, puede mantenerse en el tiempo, con la misma fundamentación y principios bioéticos: justicia, beneficencia, autonomía y no maleficencia.

\section{Consideraciones finales}

Las discapacidades presentan un incremento sostenido en el tiempo, por lo que la inclusión de las personas con deficiencias debe estar presente en todos los ámbitos de la vida, considerando los cambios que deben generarse bajo el modelo social de discapacidad. La salud y educación son áreas en las cuales las personas con discapacidad se encuentran más desprotegidas, o en condición de vulnerabilidad, por lo que se requiere permanentemente la evaluación de su situación particular. Por eso se ha generado un alto número de instancias, a partir de organismos internacionales como la ONU, para promover la inclusión de las personas con discapacidad con base en los derechos humanos.

Además de las iniciativas de la ONU, en la legislación chilena hay normativas y políticas que favorecen la integración de los estudiantes con discapacidad en la educación superior, de modo que logren una vida plena, particularmente luego que se conviertan en profesionales. Estas normativas promueven principios bioéticos fundamentales, como la autonomía y la justicia. El respeto a la dignidad de los estudiantes, como seres humanos, es también un principio que promueve la legislación y que las instituciones de educación superior deben incorporar para facilitar la inclusión.

Sin embargo, el ingreso de las personas con discapacidad a la educación superior no está exento de situaciones en las que se vulneran los principios bioéticos declarados internacionalmente. Todavía hay conflictos que siguen sin resolverse completamente, incluso con respecto a los currículos que están instalados en la formación universitaria. Se reconoce que existen esfuerzos emergentes centrados en abordar la discapacidad, sin embargo tales esfuerzos requieren cambios sustanciales y permanentes, apoyados en políticas educativas que consideren las necesidades individuales de los estudiantes con discapacidad, particularmente en las carreras de la salud. Por ahora lo que se observa es la inexistencia de directrices concretas sobre cómo formar profesionales de la salud con discapacidad, y esto es un dilema ético que demanda el análisis permanente. Se declara que existen políticas de inclusión, pero la instalación de dichas políticas es algo que requiere años.

El artículo analizó una situación de conflicto bioético en el ámbito académico a través de una metodología de trabajo que propone cursos de acción para la toma de decisiones. Mediante el análisis realizado, fue posible reflexionar sobre el proceso de deliberar y elaborar recomendaciones que consideren los principios fundamentales de la bioética. Con base en este análisis y en la literatura sobre el tema de la educación inclusiva, se concluye que son necesarios ajustes en el currículo de enfermería, de modo que se pueda contar con estrategias de intervención que respeten la dignidad humana de los estudiantes con discapacidad.

Además, se requieren políticas institucionales que generen cambios a partir de la toma de conciencia por parte de todos los integrantes de la comunidad universitaria. Es necesario que dichos cambios involucren una perspectiva bioética, puesto que las declaraciones universales de derechos humanos, que se encuentran presentes en la mayoría de las normativas actuales de la educación superior, están vinculadas estrechamente con los principios bioéticos. Se requiere también que los académicos que forman profesionales de enfermería tengan capacitación y formación para realizar cambios en los planes de estudio y principalmente para considerar a los estudiantes con discapacidad desde una perspectiva ética, teniendo presente sus particularidades como seres humanos. 


\section{Referencias}

1. Gil Obando L, López López A, Avila Rendón C, Criollo Castro C, Canchala Obando A, Serrato Medoza M. Discapacidad y su relación con variables sociodemográficas y clínicas en adultos de San Juan de Pasto, Nariño, Colombia. Univ Salud [Internet]. 2016 [acesso 6 abr 2021];18(3):505-13. DOI: 10.22267/rus.161803.55

2. Meléndez Rojas RE. Las políticas públicas en materia de discapacidad en América Latina y su garantía de acceso a una educación inclusiva de calidad. Actualidades Investigativas en Educación [Internet]. 2019 [acesso 6 abr 2021];19(2):1-26. DOI: 10.15517/aie.v19i2.36916

3. Organización Mundial de la Salud. Informe mundial sobre la discapacidad [Internet]. Ginebra: OMS; 2011 [acesso 6 abr 2021]. Disponível: https://bit.ly/3tf9k4D

4. Organización Mundial de la Salud. Op. cit. p. 7.

5. Feito Grande L. Fundamentos de bioética, de Diego Gracia. Bioética \& Debat [Internet]. 2011 [acesso 6 abr 2021];17(64):8-11. Disponível: https://bit.ly/3ebcuCa

6. Torralba Roselló F. Historia de la bioética: propuesta de principios europeos de bioética [Internet]. Barcelona: Institut Borja de Bioètica; 2021 [acesso 6 abr 2021]. Disponível: https://bit.ly/33bU6TC

7. Torralba Roselló F. Op. cit. p. 4.

8. Organización Mundial de la Salud. Op. cit. p. 8.

9. Howlin F, Halligan P, O'Toole. Development and implementation of a clinical needs assessment to support nursing and midwifery students with a disability in clinical practice: Part 1. Nurse Educ Pract [Internet]. 2014 [acesso 6 abr 2021];14(5): 557-64. DOI: 10.1016/j.nepr.2014.07.003

10. Universidad de Chile. Inclusión de estudiantes en situación de discapacidad en la Universidad de Chile: un compromiso con la equidad [Internet]. Santiago de Chile: Universidad de Chile; 2016 [acesso 6 abr 2021]. Disponível: https://bit.ly/3nMXf5B

11. Velarde-Lizama V. Los modelos de la discapacidad: un recorrido histórico. Rev Empresa Humanismo [Internet]. 2012 [acesso 6 abr 2021];15(1):115-36. Disponível: https://bit.ly/3uhlBXw

12. Brogna P, editora. Visiones y revisiones de la discapacidad. México DF: Fondo de Cultura Económica; 2009.

13. Palacios A. El modelo social de discapacidad: orígenes, caracterización y plasmación en la Convención Internacional sobre los Derechos de las Personas con Discapacidad [Internet]. Madrid: Cinca; 2008 [acesso 12 abr 2021]. Disponível: https://bit.ly/3ecOH6M

14. Palacios A. Op. cit. p. 123.

15. Aguilar Villanueva LF, editor. La implementación de las políticas. México DF: Miguel Ángel Porrúa Grupo Editorial; 1993.

16. Chile. Ley de Integración Social de las Personas con Discapacidad № 19.284 [Internet]. Santiago de Chile, 1994 [acesso 12 abr 2021]. Disponível: https://bit.ly/3h01qd0

17. Organización de las Naciones Unidas. Convención sobre los Derechos de las Personas con Discapacidad [Internet]. Ginebra: ONU; 2006 [acesso 12 abr 2021]. Disponível: https://bit.ly/3gURsJW

18. Organización de las Naciones Unidas. 2006. p. 5.

19. Organización de las Naciones Unidas. 2006. p. 20.

20. Melero Aguilar N, Moriña A, Perera VH. Acciones del profesorado para una práctica inclusiva en la universidad. Rev Bras Educ [Internet]. 2019 [acesso 6 abr 2021];24. DOI: 10.1590/s1413-24782019240016

21. Pérez-Castro J. La inclusión de los estudiantes con discapacidad en dos universidades públicas mexicanas. Innov Educ [Internet]. 2019 [acesso 6 abr 2021];19(79):145-70. Disponível: https://bit.ly/3gXhBaY

22. Chile. Ministerio de Planificación. Ley 20.422. Establece normas sobre igualdad de oportunidades e inclusión social de personas con discapacidad [Internet]. Santiago de Chile; 2010 [acesso 12 abr 2021]. Disponível: https://bit.ly/3gYSIfa

23. Chile. Servicio Nacional de la Discapacidad. Política Nacional para la Inclusión Social de las Personas con Discapacidad 2013-2020 [Internet]. Santiago de Chile: Ministerio de Desarrollo Social; 2013 [acesso 12 abr 2021]. Disponível: https://bit.ly/3vAZdsw 
24. Smeltzer S, Mariani B, Ross JG, de Mange Ep, Meakim C, Briderle E et al. Persons with disability: their experiences as standarized patients in a undergraduate nursing program. Nurs Educ Perspect [Internet]. 2015 [acesso 6 abr 2021];36(6):398-400. DOI: 10.5480/15-1592

25. Carroll SM. Inclusion of people with physical disabilities in nursing education. J Nurs Educ [Internet]. 2004 [acesso 6 abr 2021];43(5):207-12. Disponível: https://bit.ly/33ekHiR

26. Betz CL, Smith KA, Bui K. A survey of California nursing programs: admission and accomodation policies for students with disabilities. J Nurs Educ [Internet]. 2012 [acesso 6 abr 2021];51(12):676-84. DOI: 10.3928/01484834-20121112-01

27. Milsom A. Creating positive school experiences for students with disabilities [Internet]. Arlington: Reading Rockets; 2006 [acesso 6 abr 2021]. Disponível: https://bit.ly/3ebRuvd

28. Shpigelman CN, Zlotnick C, Brand R. Attitudes toward nursing students with disabilities: promoting social inclusion. J Nurs Educ [Internet]. 2016 [acesso 6 abr 2021];55(8):441-9. DOI: 10.3928/01484834-20160715-04

29. Tee $S$, Cowen $M$. Supporting students with disabilities: promoting understanding amongst mentors in practice. Nurse Educ Pract [Internet]. 2012 [acesso 6 abr 2021];12(1):6-10. DOI: 10.1016/j.nepr.2011.03.020

30. Mancussi e Faro AC, Gusmai FL. Educação inclusiva em enfermagem: análise das necessidades de estudantes. Rev Esc Enferm USP [Internet]. 2013 [acesso 6 abr 2021];47(1):225-30. DOI: 10.1590/ S0080-62342013000100029

31. Shpigelman CN, Zlotnick C, Brand R. Attitudes toward nursing students with disabilities: promoting social inclusion. J Nurs Educ [Internet]. 2016 [acesso 6 abr 2021];55(8):441-9. DOI: 10.3928/01484834-20160715-04

32. Foucault M. Los anormales. México DF: Fondo de Cultura Económica; 2008.

33. Beca Infante JP. La toma de decisiones en ética clínica [Internet]. Santiago de Chile: Universidad del Desarrollo; 2011 [acesso 6 abr 2021]. Disponível: https://bit.ly/3aZ7Fd4

34. Siurana Aparisi JC. Los principios de la bioética y el surgimiento de una bioética intercultural. Veritas [Internet]. 2010 [acesso 6 abr 2021];22:121-57. DOI: 10.4067/S0718-92732010000100006

35. Carrera Carrera J. Los fundamentos de la bioética, de H. Tristam Engelhardt. Bioética \& Debat [Internet]. 2011 [acesso 3 maio 2021];17(64):12-6. Disponível: https://bit.ly/3eRY1dw

36. Mir JT, Alibes EA. Principios de ética biomédica, de Tom L. Beauchamp y James F Childress. Bioética \& Debat [Internet]. 2011[acesso 6 abr 2021];17(64):1-7. 